TRANSACTIONS OF THE

AMERICAN MATHEMATICAL SOCIETY

Volume 365, Number 2, February 2013, Pages 837-859

S 0002-9947(2012)05612-1

Article electronically published on September 19, 2012

\title{
WEAK FUBINI PROPERTY AND INFINITY HARMONIC FUNCTIONS IN RIEMANNIAN AND SUB-RIEMANNIAN MANIFOLDS
}

\author{
FEDERICA DRAGONI, JUAN J. MANFREDI, AND DAVIDE VITTONE
}

\begin{abstract}
We examine the relationship between infinity harmonic functions, absolutely minimizing Lipschitz extensions, strong absolutely minimizing Lipschitz extensions, and absolutely gradient minimizing extensions in CarnotCarathéodory spaces. Using the weak Fubini property we show that absolutely minimizing Lipschitz extensions are infinity harmonic in any sub-Riemannian manifold.
\end{abstract}

\section{INTRODUCTION}

In this paper we study the relationships between absolutely minimizing Lipschitz extensions and infinity harmonic functions in Riemannian manifolds and CarnotCarathéodory spaces. In the classic Euclidean setting, infinity harmonic functions are the viscosity solutions of the infinity Laplace equation

$$
0=\Delta_{\infty} u=\sum_{i, j=1}^{n} u_{x_{i}, x_{j}} u_{x_{i}} u_{x_{j}}
$$

On the other hand, given $Y \Subset X \subset \mathbb{R}^{n}$ and a Lipschitz function $F: Y \rightarrow \mathbb{R}$, an absolutely minimizing Lipschitz extension (AMLE) of $F$ is a Lipschitz function $u: X \rightarrow \mathbb{R}$ such that $u=F$ on $Y$ and the Lipschitz constant of $u$

$$
\operatorname{Lip}(u, U):=\sup _{x, y \in U, x \neq y} \frac{|u(x)-u(y)|}{|x-y|}
$$

is minimal for any open set $U \Subset X \backslash Y$.

We are particularly interested in the case where $Y=\partial \Omega$ and $X=\bar{\Omega}$ for some bounded domain $\Omega \subset \mathbb{R}^{n}$. If this is the case, the AMLE of $g$ on $\bar{\Omega}$ is exactly the viscosity solution of

$$
\left\{\begin{aligned}
\Delta_{\infty} u=0 & \text { in } \Omega \\
u=g & \text { on } \partial \Omega
\end{aligned}\right.
$$

see e.g. [13.

The concept of an absolutely minimizing Lipschitz extension makes sense in any metric space; such functions exist and are uniquely determined by their boundary values in any length space (see 22] and [14]). On the other hand, the definition of infinity harmonic functions can be considered in manifolds where we have identified a way to define second-order derivatives. The two main examples of the manifolds

Received by the editors December 15, 2010 and, in revised form, April 22, 2011.

2010 Mathematics Subject Classification. Primary 53C17, 22E25, 35H20, 53C22.

Key words and phrases. Absolutely minimizing Lipschitz extension, infinity Laplace equation, Riemannian manifolds, Carnot-Carathéodory spaces. 
under consideration are Riemannian spaces and Carnot-Carathéodory (also called sub-Riemannian) spaces, both of which are length spaces endowed with their natural metric.

We recall briefly what sub-Riemannian geometries are (see [4, 17]). Let $\left\{X_{1}(x), \ldots, X_{m}(x)\right\}$ be a family of smooth vector fields on $\mathbb{R}^{n}\left(C^{1}\right.$-regularity would be enough for our purposes) and set

$$
\mathcal{X}_{x}:=\operatorname{Span}\left\{X_{1}(x), \ldots, X_{m}(x)\right\} \quad \text { and } \quad \mathcal{X}:=\left\{(x, v) \mid x \in \mathbb{R}^{n}, v \in \mathcal{X}_{x}\right\}
$$

Definition 1.1. A sub-Riemannian structure in $\mathbb{R}^{n}$ is a triple

$$
\left(\mathbb{R}^{n}, \mathcal{X},\langle\cdot, \cdot\rangle_{g}\right)
$$

where $\langle\cdot, \cdot\rangle_{g}$ is a Riemannian metric defined on $\mathcal{X}$ by requiring that the vector fields $\left\{X_{1}, \ldots, X_{m}\right\}$ are orthonormal.

An absolutely continuous curve $\gamma:[0, T] \rightarrow \mathbb{R}^{n}$ is called horizontal if $\dot{\gamma}(t) \in \mathcal{X}_{\gamma(t)}$, a.e. $t \in[0, T]$, i.e. if there exists $\alpha(t)=\left(\alpha_{1}(t), \ldots, \alpha_{m}(t)\right)$ measurable functions such that

$$
\dot{\gamma}(t)=\sum_{i=1}^{m} \alpha_{i}(t) X_{i}(\gamma(t)), \quad \text { a.e. } t \in[0, T] .
$$

We set $|\dot{\gamma}(t)|_{g}=\langle\dot{\gamma}(t), \dot{\gamma}(t)\rangle_{g}^{\frac{1}{2}}$ and define the length-functional

$$
l(\gamma)=\int_{0}^{T}|\dot{\gamma}(t)|_{g} d t=\int_{0}^{T} \sqrt{\alpha_{1}^{2}(t)+\cdots+\alpha_{m}^{2}(t)} d t .
$$

According to the previous notation we write

$$
|v|_{\mathcal{X}}=\left(\sum_{i=1}^{m} v_{i}^{2}\right)^{1 / 2}
$$

for any horizontal vector $v=\sum_{i=1}^{m} v_{i} X_{i}(x) \in \mathcal{X}_{x}$.

Once having defined the length-functional, we can introduce the following distance:

$$
d(x, y):=\inf \{l(\gamma) \mid \gamma \text { horizontal curve joining } x \text { to } y\} .
$$

Whenever the Hörmander condition is satisfied (i.e. the Lie algebra associated to $\mathcal{X}$ generates at any point the whole of $\mathbb{R}^{n}$ ) this distance is finite, continuous with respect to the Euclidean topology, and minimizing geodesics exist but, in general, they are not unique, even locally.

Several approaches have been used to get the equivalence between infinity harmonic funtions and AMLEs in different settings. We present a metric approach, based on the notion of strong absolutely minimizing Lipschitz extensions (SAMLEs) recently introduced by Juutinen and Shanmugalingam in [15. In their paper the equivalence between AMLE and SAMLE is proved in spaces where a weak Fubini property holds. One of our main results is Theorem 3.4 where we prove that such a property holds in any Carnot-Carathéodory space, thus establishing the equivalence between AMLE and SAMLE in this setting.

The paper is organized as follows. In Section 2 we discuss AMLEs in a general metric setting. We recall the notion of SAMLEs, the weak Fubini property and various results proved in [15. In Section 3 we prove the validity of the weak Fubini property in general sub-Riemannian manifolds. We first show the result in the 
particular case of Riemannian manifolds. Then we give the proof for Carnot groups and the general sub-Riemannian case. In Section 4 we recall various definitions and properties regarding the infinity Laplace equation and infinity harmonic functions in sub-Riemannian manifolds. We discuss the notion of absolutely gradient minimizing extensions (AGMEs), which is known to be equivalent to infinity harmonic functions in Riemannian manifolds and Carnot groups (see [3, 25]). However, in general sub-Riemannian manifolds only one implication is known: any AGME is infinity harmonic. We then show that AGMEs are equivalent to SAMLEs, which allows us to derive our main result: in a general Carnot-Carathéodory space any AMLE is an infinity harmonic function. A first application of this result is related to the theory of the tug-of-war, introduced by Y. Peres, O. Schramm, S. Sheffield and D.B. Wilson in 22. They prove that the limit of the values of suitable tug-of-war stochastic games is the unique AMLE of a given Lipschitz function $g$, in any length space. In particular, they deduce that such a value function is also infinity harmonic in the Euclidean case. By applying our result, we can conclude that this limit of value functions of tug-of-war games is infinity harmonic also in sub-Riemannian manifolds.

\section{AMLEs, SAMLES AND THE WEAK FUBINI PROPERTY}

The problem of finding a Lipschitz extension of some given Lipschitz function $F: Y \subset \mathbb{R}^{n} \rightarrow \mathbb{R}$, has been studied extensively. See for example [2, 8]. Given a metric space $(X, d)$, a proper subset $Y$ of $X$, and a Lipschitz function $F: Y \rightarrow \mathbb{R}$, we are interested in finding a "good" Lipschitz function, extending $F$ to the whole $X$. Before giving the definitions, we recall that the Lipschitz constant of $F$ in a subset $Y$ is defined as

$$
\operatorname{Lip}(F, Y)=\sup _{\substack{x, y \in Y \\ x \neq y}} \frac{|F(x)-F(y)|}{d(x, y)} .
$$

Given a metric space $(X, d)$, a proper subset $Y$ of $X$ and a Lipschitz function $F: Y \rightarrow \mathbb{R}$, finding a minimal Lipschitz extension of $F$ on $X$ requires finding a Lipschitz function $u: X \rightarrow \mathbb{R}$ such that

$$
\operatorname{Lip}(F, Y)=\operatorname{Lip}(u, X) .
$$

Minimal Lipschitz extensions always exist. McShane and Whitney found the two following minimal extensions (see [16, 26]):

$$
u_{1}(x)=\inf _{y \in Y}\{F(y)+\operatorname{Lip}(F, Y) d(x, y)\}
$$

and

$$
u_{2}(x)=\sup _{y \in Y}\{F(y)-\operatorname{Lip}(F, Y) d(x, y)\} .
$$

Hence minimal Lipschitz extensions are, in general, not unique.

Definition 2.1. Let $(X, d)$ be a metric space and $Y$ a proper subset of $X$. Given a Lipschitz function $F: Y \rightarrow \mathbb{R}$, we say that $u: X \rightarrow \mathbb{R}$ is an absolutely minimizing Lipschitz extension (AMLE) of $F$ on $X$ when

(i) $u$ is a minimal Lipschitz extension of $F$ on $X$,

(ii) for any open set $U \Subset X \backslash Y$,

$$
\operatorname{Lip}(u, U)=\operatorname{Lip}(u, \partial U) .
$$


We say that a function $u$ is AMLE in an open set $\Omega \subset \mathbb{R}^{n}$ if it is AMLE of its boundary datum, i.e. if $u \in \operatorname{Lip}(\bar{\Omega})$ and (7) holds, for any $U \Subset \Omega$.

In the Euclidean setting, it is well known that it is possible to characterize AMLEs using a comparison with cones $(2,8])$. Champion and De Pascale have shown in 7 how to extend this characterization to any length space $(X, d)$, by using suitable metric cones.

Definition 2.2. Let $(X, d)$ Modified be a length space and $\Omega$ be a proper and open subset of $X$. We say that the function $u: \bar{\Omega} \rightarrow \mathbb{R}$ satisfies comparison with metric cones from above if, for any $z \in X, a \in \mathbb{R}, b \geq 0$ and $U \subset \Omega$ open, the implication

$$
u(x) \leq a+b d(x, z) \text { on } \partial(U \backslash\{z\}) \Rightarrow u(x) \leq a+b d(x, z) \text { on } \bar{U}
$$

holds. We say that the function $u$ satisfies comparison with metric cones from below if, for any $z, a, b$ and $U$ as before, we have

$$
u(x) \geq a-b d(x, z) \text { on } \partial(U \backslash\{z\}) \Rightarrow u(x) \geq a-b d(x, z) \text { on } \bar{U} .
$$

The function $u$ satisfies comparison with metric cones if $u$ satisfies comparisons from above and below. Note that a function $u$ satisfies comparison from below exactly when $-u$ satisfies comparison from above.

Remark 2.3. For any $z \in X, b \geq 0$ and $a \in \mathbb{R}$, the function $\varphi(x)=a \pm b d(x, z)$ in the above definition is called a metric cone.

Theorem 2.4 ([7). Let $(X, d)$ be a length space and $\Omega \subset \mathbb{R}^{n}$ be open and bounded. Then $u: \bar{\Omega} \rightarrow \mathbb{R}$ is an AMLE if and only if u satisfies the comparison with metric cones in $\Omega$.

Recall that a length space is a metric space where the distance between any pair of points is the infimum of the lengths of continuous arcs joining them. Riemannian and sub-Riemannian manifolds are length spaces.

The existence of AMLEs in general length spaces was first shown by Juutinen in 14, while the uniqueness was proved by Peres, Schramm, Sheffield and Wilson in 22. by using the fact that AMLEs arise as limits of value functions of random tugof-war games when the step tends to zero. In particular, they prove the following.

Theorem 2.5 (22]). Let $(X, d)$ be a length space and $Y$ be a proper subset of $X$. For any given Lipschitz function $F: Y \rightarrow \mathbb{R}$, there exists a unique AMLE of $F$ on $X$.

Next, we introduce the notion of strong absolutely minimizing Lipschitz extensions. Let $U$ be an open subset in a metric measure space $(X, d, \mu)$ and $F: U \rightarrow \mathbb{R}$ be a function. For a fixed point $x \in U$, the local Lipschitz constant of $F$ at the point $x$ is given by

$$
\operatorname{Lip} F(x)=\lim _{R \rightarrow 0^{+}} \sup _{y \in B_{R}(x) \backslash\{x\}} \frac{|F(x)-F(y)|}{d(x, y)},
$$

where $B_{R}(x)$ is the ball of radius $R$ and center $x$. The local Lipschitz constant of a function $F$ on an open set $U \subset X$ is defined as

$$
\widehat{\operatorname{Lip}}(F, U)=\mu \text { - } \underset{x \in U}{\operatorname{essup}} \operatorname{Lip} F(x) .
$$

Here, $\mu$ denotes the "natural" measure on the space $X$. For Riemannian manifolds, this has to be understood as the volume measure induced by the Riemannian 
structure; when dealing with a sub-Riemannian structure defined on $\mathbb{R}^{n}, \mu$ will instead denote the Lebesgue measure $\mathcal{L}^{n}$. The measure $\mu$ will always be understood and frequently omitted.

Definition 2.6. Let $(X, d, \mu)$ be a metric measure space. Given a Lipschitz function $F: Y \rightarrow \mathbb{R}^{n}$ with $Y \subset X$, we say that a Lispchitz function $u: X \rightarrow \mathbb{R}^{n}$ is a strong absolutely minimizing Lipschitz extension (SAMLE) of $F$ on $X$ if $u=F$ on $Y$ and, for any $U \Subset X \backslash Y$ and for any $v \in \operatorname{Lip}(\bar{U})$ with $u=v$ on $\partial U$, we have

$$
\widehat{\operatorname{Lip}}(u, U) \leq \widehat{\operatorname{Lip}}(v, U) \text {. }
$$

Note that we always have $\widehat{\operatorname{Lip}}(u, U) \leq \operatorname{Lip}(u, U)$. The opposite inequality depends on the structure of the set. In fact, if $U$ is not geodesically convex, it is possible to give examples where $\widehat{\operatorname{Lip}}(u, U)<\operatorname{Lip}(u, U)$ (see Example 4.6 below).

Nevertheless, in many cases there is no difference between minimizing $\widehat{\operatorname{Lip}}(u, U)$ or $\operatorname{Lip}(u, U)$. To study when this is the case we need to introduce the weak Fubini property, which is the main object under investigation in this paper.

Consider a metric measure space $(X, d, \mu)$. Given a family $\Gamma$ of curves in $X$, the set of admissible metrics is

$$
M(\Gamma)=\left\{\rho: \rho \geq 0 \text { is Borel and } \int_{\gamma} \rho \geq 1 \text { for all } \gamma \in \Gamma\right\} .
$$

The modulus of the curve family $\Gamma$ is given by

$$
\operatorname{Mod} \Gamma=\inf \left\{\int_{X} \rho d \mu: \rho \in M(\Gamma)\right\} .
$$

Note that if two curve families satisfy $\Gamma_{1} \subset \Gamma_{2}$, then their moduli satisfy Mod $\Gamma_{1} \leq$ $\operatorname{Mod} \Gamma_{2}$.

Definition 2.7. Let $(X, d, \mu)$ be a metric measure space. We say that $X$ satisfies the weak Fubini property if there exists $C>0$ and $\tau_{0}>0$ such that, for any $0<\tau \leq \tau_{0}$ and $B_{1}, B_{2}$ balls with

$$
d\left(B_{1}, B_{2}\right)>\tau \max \left\{\operatorname{diam}\left(B_{1}\right), \operatorname{diam}\left(B_{2}\right)\right\},
$$

we have

$$
\operatorname{Mod} \Gamma\left(B_{1}, B_{2}, \tau\right)>0,
$$

where the family $\Gamma=\Gamma\left(B_{1}, B_{2}, \tau\right)$ consists of all curves in $X$, joining $B_{1}$ and $B_{2}$ and such that $l(\gamma) \leq d\left(B_{1}, B_{2}\right)+C \tau$.

Remark 2.8. The property, given by Definition 2.7, is called weak Fubini since it can be related to the existence of a Fubini-type decomposition for the measure $\mu$. Let us clarify this in the Euclidean space $\mathbb{R}^{n}$ endowed with the Lebesgue measure $\mathcal{L}^{n}$. For some fixed $\tau>0$ let us consider a segment $\bar{\gamma} \in \Gamma:=\Gamma\left(B_{1}, B_{2}, \tau\right)$ and let $U$ be a neighbourhood of $\bar{\gamma}$ such that $U \subset \bigcup\{\gamma: \gamma \in \Gamma\}$. Let $\bar{x} \in B_{1}$ be the first endpoint of $\bar{\gamma}$ and $\Pi$ be the affine plane through $\bar{x}$ orthogonal to $\bar{\gamma}$; set $V:=B_{1} \cap \Pi$. Then we can decompose $\mathcal{L}^{n}$ as $\left.d \mathcal{L}^{n}\right|_{U}=\left.d \mathcal{H}^{n-1}\right|_{V} \oplus d t$ and, by the Fubini Theorem, for any $\rho \in M(\Gamma)$, we have

$$
\int_{X} \rho d \mathcal{L}^{n} \geq \int_{U} \rho d \mathcal{L}^{n}=\int_{V}\left(\int_{\gamma_{x}} \rho d t\right) d \mathcal{H}^{n-1}(x) \geq \mathcal{H}^{n-1}(V)>0,
$$

where $\gamma_{x}$ denotes the segment (belonging to $\Gamma$ ) parallel to $\bar{\gamma}$ and with endpoint $x \in V$. Hence the weak Fubini property holds. 
Using the weak Fubini property, Juutinen and Shanmugalingam proved the following equivalence.

Theorem $2.9([15])$. Let $(X, d, \mu)$ be a metric measure space. If $(X, d)$ is a length space and the weak Fubini property holds, then $u$ is an AMLE if and only if $u$ is a SAMLE.

Our goal is to prove that the weak Fubini property holds in any sub-Riemannian manifold, so that Theorem 2.9 applies to such structures.

\section{ThE WEAK Fubini PROPERTY IN SUB-RIEMANNIAN MANIFOLDS}

The proof of the validity of the weak Fubini property in general sub-Riemannian manifolds is somewhat technical. We show the result first in the particular cases of Riemannian manifolds and Carnot groups, where the proofs are easier.

Theorem 3.1. Let $(M, d, \mu)$ be an n-dimensional Riemannian manifold endowed with the geodesic distance $d$ and the natural volume measure $\mu$. Then the weak Fubini property holds.

Proof. Set $C=1$ and fix $\tau>0$. Consider $B_{1}, B_{2}$ as in Definition 2.7. Fix $x \in B_{1}$ and $y \in B_{2}$ such that $d(x, y) \leq d\left(B_{1}, B_{2}\right)+\tau / 2$. Write $d=d(x, y)$ and consider a quasi-geodesic $\gamma:[0, d+\epsilon] \rightarrow M$, parametrized by arc length, with $\gamma(0)=x$ and $\gamma(d+\epsilon)=y$. We may assume that $\operatorname{Im} \gamma \subset M$ is an embedded submanifold and that $l(\gamma)=d+\epsilon \leq d\left(B_{1}, B_{2}\right)+\tau / 2$.

Our strategy is to build a suitable subfamily $A$ of all the admissible curves $\Gamma:=\Gamma\left(B_{1}, B_{2}, \tau\right)$ and show that $\operatorname{Mod}(A)>0$. Denote by $N \gamma$ the normal bundle (with rank $n-1$ ) to the submanifold $\gamma$,

$$
N \gamma=\left\{(\gamma(t), w) \mid w \in N_{\gamma(t)} \gamma \subset T_{\gamma(t)} M, t \in[0, d+\epsilon]\right\},
$$

and consider the exponential map

$$
\begin{aligned}
& \exp : \quad N \gamma \mapsto \mathbb{R}^{n} \\
& (\gamma(t), w) \mapsto \exp (w)(\gamma(t)) .
\end{aligned}
$$

It is well known (see e.g. [20, Ch. 7, Prop. 26]) that $\gamma$ has a normal neighbourhood $U$ in $M$; i.e. exp : $Z \rightarrow U$ is a diffeomorphism between $U$ and an open set $Z \subset N \gamma$ with the property that $(\gamma(t), 0) \in Z$ for any $t$. It is not restrictive to assume that $Z$ is diffeomorphic to $[0, d+\epsilon] \times V$ for some open set $V \subset \mathbb{R}^{n-1}$. Denote by $I:[0, d+\epsilon] \times V \rightarrow Z$ such a diffeomorphism, which we can assume to satisfy $I(t, 0)=(\gamma(t), 0)$. The composition

$$
F=\exp \circ I:[0, d+\epsilon] \times V \rightarrow U
$$

is a diffeomorphism and satisfies $F(t, 0)=\exp (\gamma(t), 0)=\gamma(t)$. Without loss of generality, we may assume that

$$
\inf \{|\operatorname{det} J F(t, v)|:(t, v) \in[0, d+\epsilon] \times V\}=k>0,
$$

where by $J F$ we mean the Jacobian matrix of $F$ with respect to $(t, v)$. For any $v \in V$ define the curve $\gamma_{v}(t)=F(t, v)$. Recall that $\gamma_{0}=F(\cdot, 0)=\gamma$. The subfamily of all the admissible curves we are going to consider is $A=\left\{\gamma_{v}:=F(\cdot, v) \mid v \in V\right\} \subset \Gamma$. In fact, possibly restricting $V$ and $U$, we can assume that

$$
l\left(\gamma_{v}\right) \leq l(\gamma)+\frac{\tau}{2}, \quad \gamma_{v}(0) \in B_{1} \quad \text { and } \quad \gamma_{v}(d+\epsilon) \in B_{2}
$$


so that $\gamma_{v} \in \Gamma$ for any $v \in V$. Since $\left|\dot{\gamma}_{0}(t)\right|=|\dot{\gamma}(t)|=1$, we can always choose $V$ sufficiently small in order to have $\left|\dot{\gamma}_{v}(t)\right| \leq 2$ for any $(t, v) \in[0, d+\epsilon] \times V$ (in fact $F(t, v)$ is a diffeomorphism, so $\dot{\gamma}_{v} \rightarrow \dot{\gamma}$, as $\left.v \rightarrow 0\right)$.

Consider a Borel positive function $\rho: M \rightarrow \mathbb{R}$ with $\int_{\gamma} \rho \geq 1$ for any $\gamma \in \Gamma$. A change of variable gives

$$
\begin{aligned}
\int_{M} \rho d \mu & \geq \int_{U} \rho d \mu \\
& =\int_{[0, d+\epsilon] \times V} \rho(F(t, v))|\operatorname{det} J F(t, v)| d \mathcal{L}^{n-1}(v) d t \\
& \geq k \int_{V}\left(\int_{0}^{d+\epsilon} \rho\left(\gamma_{v}(t)\right) d t\right) d \mathcal{L}^{n-1}(v) .
\end{aligned}
$$

Given $\gamma_{v} \in \Gamma$ we can observe that

$$
1 \leq \int_{\gamma_{v}} \rho=\int_{0}^{d+\epsilon} \rho\left(\gamma_{v}(t)\right)\left|\dot{\gamma}_{v}(t)\right| d t \leq 2 \int_{0}^{d+\epsilon} \rho\left(\gamma_{v}(t)\right) d t
$$

and conclude

$$
\operatorname{Mod}(A)=\inf \left\{\int_{M} \rho d \mu \mid \rho \text { admissible for } A\right\} \geq \frac{k}{2} \mathcal{L}^{n-1}(V)>0,
$$

since $V$ is open and nonempty in $\mathbb{R}^{n-1}$. Hence $\operatorname{Mod}(\Gamma)>0$, as desired.

To show that the weak Fubini property holds in Carnot groups we extend the previous argument. We briefly recall that a Carnot group $\mathbb{G}$ is a Carnot-Carathéodory space endowed with a group operation - and a one-parameter group of dilations $\left(\delta_{s}\right)_{s \geq 0}$ such that

$$
\left\|\delta_{s}(x)\right\|_{C C}=s\|x\|_{C C}, \quad \text { for any } s \geq 0
$$

where $\|x\|_{C C}=d(x, 0)$ is the Carnot-Carathéodory norm defined on $\mathbb{G}$. We recall also that a Carnot group of finite step $k$ has a stratified Lie algebra $g$ with grading $g=\bigoplus_{i=1}^{k} V_{i}$. Since $k$ is finite, the exponential map between $g$ and $\mathbb{G}$ is always assumed to be the identity (after possibly applying an appropriate diffeomorphism). Vector fields in $V_{i}$ have order $i$. The full gradient of a function $u$ therefore consists of derivatives of different orders. The horizontal gradient is the projection of the gradient onto $V_{1}$. We shall denote the second-order terms of the gradient by $T u$ and we note that it is the projection of the gradient onto $V_{2}$. For more complete details concerning Carnot groups, we direct the interested reader to [12] and the references therein.

Next we show the validity of the weak Fubini property in Carnot groups. The proof will serve as the basis for the sub-Riemannian case, which is necessarily more technical.

We begin with a technical lemma, which will also be a key point in the proof of the general result.

Lemma 3.2. Let $\mathcal{X}=\left\{X_{1}(x), \ldots, X_{m}(x)\right\}$ be a family of smooth vector fields in $\mathbb{R}^{n}$ satisfying the Hörmander condition and let $B_{1}, B_{2}$ be balls in $\mathbb{R}^{n}$ with positive distance. For any $\tau>0$ there esists a smooth horizontal curve $\gamma$ between $x \in B_{1}$ and $y \in B_{2}$ such that

$$
l(\gamma) \leq d\left(B_{1}, B_{2}\right)+\frac{\tau}{2}
$$


Proof. By the Hörmander condition, there exists a horizontal curve $\gamma_{0}:[0, T] \rightarrow \mathbb{R}^{n}$ between $\overline{B_{1}}$ and $\overline{B_{2}}$, which realizes the distance. Note that in general $\gamma_{0}(0) \in \partial B_{1}$ and $\gamma_{0}(T) \in \partial B_{2}$. Nevertheless, since the distance is continuous, we can always find a new horizontal curve $\gamma:[0, T] \rightarrow \mathbb{R}^{n}$ such that $\gamma(0) \in B_{1}, \gamma(T) \in B_{2}$ and $l(\gamma) \leq d\left(B_{1}, B_{2}\right)+\frac{\tau}{4}$.

Let us now consider such a quasi-geodesic $\gamma$ between $B_{1}$ and $B_{2}$. Since $\gamma$ is horizontal, then

$$
\dot{\gamma}(t)=\sum_{i=1}^{m} \alpha_{i}(t) X_{i}(\gamma(t)),
$$

for some measurable function $\alpha(t)=\left(\alpha_{1}(t), \ldots, \alpha_{m}(t)\right) \in \mathbb{R}^{m}$.

Up to a reparametrization, we can assume that $\alpha_{i} \in L^{\infty}([0, T])$. For any $\tau>0$, we define a family of horizontal smooth curves $\gamma^{\tau}$ by

$$
\dot{\gamma}^{\tau}(t)=\sum_{i=1}^{m} \alpha_{i}^{\tau}(t) X_{i}\left(\gamma^{\tau}(t)\right), \quad \gamma^{\tau}(0)=\gamma(0),
$$

where $C^{\infty}([0, T]) \ni \alpha^{\tau} \rightarrow \alpha$ in $L^{1}([0, T])$ as $\tau \rightarrow 0$ (the existence of such an $\alpha^{\tau}(t)$ is standard by convolutions).

In order to conclude the proof, it is enough to prove that $l\left(\gamma^{\tau}\right) \rightarrow l(\gamma)$ and $\gamma^{\tau}(T) \rightarrow \gamma(T)$ as $\tau \rightarrow 0$.

We first show the convergence of $l\left(\gamma^{\tau}\right)$. In fact,

$$
\begin{aligned}
\left|l\left(\gamma^{\tau}\right)-l(\gamma)\right| & =\left|\int_{0}^{T}\left\|\alpha^{\tau}(t)\right\| d t-\int_{0}^{T}\|\alpha(t)\| d t\right| \\
& \leq \int_{0}^{T}\left|\left\|\alpha^{\tau}(t)\right\|-\|\alpha(t)\|\right| d t \\
& \leq \int_{0}^{T}\left\|\alpha^{\tau}(t)-\alpha(t)\right\| d t \rightarrow 0, \quad \text { as } \tau \rightarrow 0 .
\end{aligned}
$$

The pointwise convergence of the curves can be easily proved by Gronwall's Lemma. Recalling that $\gamma(0)=\gamma^{\tau}(0)$ for any $\tau>0$, then

$$
\begin{aligned}
& \left|\gamma^{\tau}(t)-\gamma(t)\right| \leq \sum_{i=1}^{m} \int_{0}^{t}\left|\alpha_{i}^{\tau}(s) X_{i}\left(\gamma^{\tau}(s)\right)-\alpha_{i}(s) X_{i}(\gamma(s))\right| d s \\
\leq & \sum_{i=1}^{m} \int_{0}^{t}\left(\left|\alpha_{i}^{\tau}(s)-\alpha_{i}(s)\right|\left|X_{i}(\gamma(s))\right|+\left|\alpha_{i}^{\tau}(s)\right|\left|X_{i}\left(\gamma^{\tau}(s)\right)-X_{i}(\gamma(s))\right|\right) d s,
\end{aligned}
$$

where we have added $\pm \sum_{i=1}^{m} \alpha_{i}^{\tau}(s) X_{i}(\gamma(s))$. Since $\alpha_{i}$ and $X_{i}$ are smooth, they are Lipschitz and bounded in a compact set containing $\operatorname{Im}(\gamma)$. Moreover we may assume that $\left\|\alpha_{i}^{\tau}\right\|_{\infty} \leq\left\|\alpha_{i}\right\|_{\infty}$, where the bound is independent of $\tau$. Therefore we get the following estimates:

$$
\begin{aligned}
& \left|\gamma^{\tau}(t)-\gamma(t)\right| \leq C_{1} \sum_{i=1}^{m} \int_{0}^{t}\left|\alpha_{i}^{\tau}(s)-\alpha_{i}(s)\right| d s+C_{2} \int_{0}^{t}\left|\gamma^{\tau}(s)-\gamma(s)\right| d s \\
& \leq C_{1} \sum_{i=1}^{m} \int_{0}^{T}\left|\alpha_{i}^{\tau}(s)-\alpha_{i}(s)\right| d s+C_{2} \int_{0}^{t}\left|\gamma^{\tau}(s)-\gamma(s)\right| d s .
\end{aligned}
$$


Noting that $C_{1} \sum_{i=1}^{m} \int_{0}^{T}\left|\alpha_{i}^{\tau}(s)-\alpha_{i}(s)\right| d s$ is constant in $t$, then, by Gronwall's Lemma, we can conclude

$$
\left|\gamma^{\tau}(t)-\gamma(t)\right| \leq C_{1} e^{C_{2} T} \sum_{i=1}^{m} \int_{0}^{T}\left|\alpha_{i}^{\tau}(s)-\alpha_{i}(s)\right| d s \rightarrow 0, \quad \text { as } \tau \rightarrow 0 .
$$

In particular, we can apply the previous convergence to $t=T$ and deduce that $\gamma^{\tau}(T) \in B_{2}$ for sufficiently small $\tau>0$.

This concludes the proof.

We can now prove the weak Fubini property in Carnot groups.

Theorem 3.3. In any Carnot group $\mathbb{G}$, endowed with the associated CarnotCarathéodory distance $d(x, y)$ and the natural volume measure $\mu$, the weak Fubini property holds.

Proof. Let $C=1, \tau>0$ be fixed and $B_{1}, B_{2}$ be as in Definition 2.7. We can consider a quasi-geodesic $\gamma$ as in Lemma 3.2, i.e. $\gamma$ smooth, horizontal and such that $l(\gamma) \leq d\left(B_{1}, B_{2}\right)+\frac{\tau}{2}$, with $x=\gamma(0) \in B_{1}$ and $y=\gamma(T) \in B_{2}$.

Since $\gamma(t)$ is horizontal and smooth, then there exists a smooth function $\alpha$ : $[0, T] \rightarrow \mathbb{R}^{m}$ with $\alpha(t)=\left(\alpha_{1}(t), \ldots, \alpha_{m}(t)\right)$ such that

$$
\dot{\gamma}(t)=\sum_{j=1}^{m} \alpha_{j}(t) X_{j}(\gamma(t)), \quad \text { for any } t \in[0, T]
$$

We can approximate in $L^{1}$ the function $\alpha$ by locally-constant functions; i.e. we can find $h:[0, T] \rightarrow \mathbb{R}^{m}$ such that:

i) There exist values

$$
0=T_{0}<T_{1}<T_{2}<\cdots<T_{q}=T
$$

such that $h(t)=h^{i}=\left(h_{1}^{i}, \ldots, h_{m}^{i}\right) \in \mathbb{R}^{m}$, whenever $t \in\left(T_{i-1}, T_{i}\right]$, with $i=1, \ldots, q$.

ii) $h_{1}^{i} \neq 0$, for any $i=1, \ldots, q$. In particular there exists $\delta>0$ such that $\left|h_{1}^{i}\right| \geq \delta$, for any $i=1, \ldots, q$.

iii) If $\eta:[0, T] \rightarrow \mathbb{G}$ is the curve defined by $\eta(0)=x$ and

$$
\dot{\eta}(t)=\sum_{j=1}^{m} h_{j}(t) X_{j}(\eta(t))
$$

then $l(\eta)<l(\gamma)+\frac{\tau}{2} \leq d\left(B_{1}, B_{2}\right)+\tau$. Moreover, as we have seen in Lemma $3.2 y^{\prime}:=\eta(T)$ is close to $y$, which implies $y^{\prime} \in B_{2}$.

Recall that we identify $\mathbb{G}$ with $\mathbb{R}^{n}$ by means of exponential coordinates. It is well known that the set

$$
\mathbb{W}:=\left\{x \in \mathbb{R}^{n} \mid x_{1}=0\right\}
$$

is a maximal subgroup of $\mathbb{G}$, which can be identified with $\mathbb{R}^{n-1}$ via the map $\mathbb{R}^{n-1} \ni$ $w \mapsto(0, w) \in \mathbb{R}^{n} \equiv \mathbb{G}$. Given a (relatively) open neighbourhood $U \subset \mathbb{W} \equiv \mathbb{R}^{n-1}$ of $x$, we define the map

$$
\begin{aligned}
\Phi: \quad & U \times[0, T] \rightarrow \mathbb{G} \equiv \mathbb{R}^{n} \\
& (w, t) \longmapsto(0, w) \cdot \eta(t) .
\end{aligned}
$$


The translated curve $\eta_{w}(t):=\Phi(w, \eta(t))$ is still smooth on $[0, T]$ and so, if $U$ is sufficiently small, it joins $w \in B_{1}$ (which is a point close to $x$ ) to a point $w \cdot y^{\prime} \in B_{2}$ and

$$
l\left(\eta_{w}\right)=l(\eta)<d\left(B_{1}, B_{2}\right)+\tau,
$$

for any $w \in \mathbb{W}$. In particular, $\eta_{w} \in \Gamma:=\Gamma\left(B_{1}, B_{2}, \tau\right)$ for any $w \in U$.

Assume for a moment that

$$
|\operatorname{det} J \Phi(w, t)| \geq \delta \text { for } \mathcal{L}^{n} \text {-a.e. }(w, t) \in \mathbb{R}^{n} .
$$

Consider a Borel function $\rho \geq 0$ on $\mathbb{G}$ such that $\int_{c} \rho \geq 1$ for any $c \in \Gamma$. In particular $\int_{\eta_{w}} \rho \geq 1$ for any $w \in U$ and therefore there exists a measurable function $i: U \rightarrow\{1, \ldots, q\}$ such that

$$
\int_{\eta_{w}^{i}} \rho \geq \frac{1}{q}, \quad \text { for any } w \in U, \quad \text { where } \eta_{w}^{i}:=\left.\eta_{w}\right|_{\left(T_{i(w)-1}, T_{i(w)}\right]} .
$$

Fix $i_{0} \in\{1, \ldots, q\}$ such that $\mathcal{L}^{n-1}\left(U_{i_{0}}\right)>0$, where $U_{i_{0}}:=\left\{w \in U \mid i(w)=i_{0}\right\}$. Therefore

$$
\begin{aligned}
\int_{\mathbb{G}} \rho d \mu & \geq \int_{\Phi\left(U_{i_{0}} \times\left(T_{i_{0}-1}, T_{i_{0}}\right)\right)} \rho d \mu \\
& =\int_{U_{i_{0}}}\left(\int_{T_{i_{0}-1}}^{T_{i_{0}}}|\operatorname{det} J \Phi(w, t)| \rho\left(\eta_{w}(t)\right) d t\right) d \mathcal{L}^{n-1}(w) \\
& \geq \int_{U_{i_{0}}} \frac{\delta}{\left\|\dot{\eta}_{w}\right\|_{\infty}}\left(\int_{T_{i_{0}-1}}^{T_{i_{0}}}\left|\dot{\eta_{w}}(t)\right| \rho\left(\eta_{w}(t)\right) d t\right) d \mathcal{L}^{n-1}(w) \\
& \geq \frac{\delta}{\|\dot{\eta}\|_{\infty}} \frac{1}{q} \mathcal{L}^{n-1}\left(U_{i_{0}}\right)
\end{aligned}
$$

and this implies that

as desired.

$$
\operatorname{Mod}(\Gamma) \geq \frac{\delta \mathcal{L}^{n-1}\left(U_{i_{0}}\right)}{q\|\dot{\eta}\|_{\infty}}>0
$$

We still need to prove that $|\operatorname{det} J \Phi(w, t)| \geq \delta$ for $\mathcal{L}^{n}$-a.e. $(w, t) \in \mathbb{R}^{n}$. Let $t \in$ $[0, T]$ be fixed. By the definition of $\Phi$, the first coordinates of $\eta$ and $\eta_{w}$ coincide: this is due to the Baker-Campbell-Hausdorff formula (see e.g., 24]) which implies that, in exponential coordinates, the group law is commutative in the first $m$ coordinates (i.e., the horizontal ones).

Therefore we can consider the map $\Phi_{t}$ defined by

$$
\mathbb{R}^{n-1} \equiv \mathbb{W} \supset U \ni w \stackrel{\Phi_{t}}{\longmapsto} \Phi(w, t) \in\left\{\eta_{1}(t)\right\} \times \mathbb{R}^{n-1} \equiv \mathbb{R}^{n-1} .
$$

Since $\frac{\partial}{\partial t} \Phi(w, t)=\dot{\eta}(t)=\sum_{j=1}^{m} h_{j}(t) X_{j}(\eta(t))$, the first component of $\frac{\partial}{\partial t} \Phi(w, t)$ is exactly $h_{1}$, because in exponential coordinates the vector fields look like

$$
X_{1}=(\underbrace{1,0, \ldots, 0}_{\in \mathbb{R}^{m}}, *, \ldots, *) \quad \ldots \quad X_{m}=(\underbrace{0, \ldots, 0,1}_{\in \mathbb{R}^{m}}, *, \ldots, *) .
$$

Therefore

$$
J \Phi(w, t)=\left(\begin{array}{cc}
\mathbf{0} & h^{1}(t) \\
J \Phi_{t}(w) & v(t)
\end{array}\right)
$$

where $\mathbf{0}$ is the null $1 \times(n-1)$-matrix and $v(t) \in \mathbb{R}^{n-1}$. This implies that $|\operatorname{det} J \Phi|=$ $\left|h_{1}(t)\right|\left|\operatorname{det} J \Phi_{t}\right|$. 
By assumption (ii), our claim will be proved once we show that

$$
\operatorname{det} J \Phi_{t}=1 \text {. }
$$

That is easy to verify. In fact, the differential of the right translation by $\eta(t)$ is an $n \times n$ lower triangular matrix $M$ with elements on the diagonal equal to 1 . Hence, it is sufficient to observe that $J \Phi_{t}$ is the $(n-1) \times(n-1)$ minor of $M$ obtained by erasing its first row and its first column to conclude that $\operatorname{det} J \Phi_{t}=1$.

We conclude this section by proving the weak Fubini property for general subRiemannian spaces.

Let $\mathcal{X}$ be a distribution on $\mathbb{R}^{n}$ satisfying the Hörmander condition. We assume that $\operatorname{dim} \mathcal{X}_{x}=m$ is independent from $x$ and that $\mathcal{X}$ is spanned by smooth vector fields $X_{1}(x), \ldots, X_{m}(x)$ satisfying the Hörmander condition. Using Lemma 3.2 it is not difficult to see that for open sets $E, F \subset \mathbb{R}^{n}$,

$$
\begin{gathered}
d(E, F)=\inf \left\{l(\gamma) \mid \gamma:[0, T] \rightarrow \mathbb{R}^{n}\right. \text { horizontal and smooth, } \\
\gamma(0) \in E, \gamma(T) \in F\} .
\end{gathered}
$$

where the length $l(\gamma)$ is the one given by (3) and $d$ is the Carnot-Carathéodory distance defined in (5).

Theorem 3.4. Let $\left(\mathbb{R}^{n}, \mathcal{X},\langle\cdot, \cdot\rangle_{g}\right)$ be a sub-Riemannian space endowed with the Carnot-Carathéodory distance defined in (5) and the natural volume measure $\mu$. Then the weak Fubini property holds.

Proof. Let $\tau>0$ be fixed and consider balls $B_{1}, B_{2}$ as in Definition 2.7. By (10) there exists a smooth horizontal curve $\gamma:[0, T] \rightarrow \mathbb{R}^{n}$ such that $\gamma(0) \in B_{1}, \gamma(T) \in$ $B_{2}$, with

$$
l(\gamma) \leq d\left(B_{1}, B_{2}\right)+\frac{\tau}{2} .
$$

We may also assume that $\gamma$ is parametrized by arclength, so that $T=l(\gamma)$. Since $\dot{\gamma}$ is smooth and $|\dot{\gamma}|=1$, then $\gamma$ is locally injective.

For the reader's convenience, we divide the proof into several steps. Our strategy is as follows: In Steps 0-3 we construct a family $A=\left\{\gamma_{x}\right\}_{x \in \Sigma_{0}}$ of horizontal Lipschitz curves $\gamma_{x}:[0, T] \rightarrow \mathbb{R}^{n}$ which are, in some sense, parallel to $\gamma$. The space of parameters $\Sigma_{0}$ is an open subset of $\mathbb{R}^{n-1}$ containing the origin. The family of curves $A$ spans a neighbourhood $U$ of $\gamma$ in $\mathbb{R}^{n}$. They can be constructed in such a way that $\gamma_{0}=\gamma$ and $\gamma_{x} \in \Gamma:=\Gamma\left(B_{1}, B_{2}, \tau\right)$ for any $x \in \Sigma_{0}$, i.e. $A \subset \Gamma$ (see Step $4)$.

We claim that $\operatorname{Mod} A>0$, which would imply $\operatorname{Mod} \Gamma>0$. To this aim, let $\rho$ be a positive Borel function on $\mathbb{R}^{n}$ such that $\int_{c} \rho \geq 1$ for any curve $c \in \Gamma$; in particular, $\int_{\gamma_{x}} \rho \geq 1$ for any $\gamma_{x} \in A$. Our explicit construction will show that the speed of any $\gamma_{x}$ is bounded away from 0 . This condition will imply that

$$
\int_{0}^{T} \rho\left(\gamma_{x}(s)\right) d s \geq C_{1}>0
$$

and, in turn, that $\int_{[0, T] \times \Sigma_{0}} \rho\left(\gamma_{x}(s)\right) d \mathcal{L}^{n}(s, x) \geq C_{2}>0$. From this information, and the fact that the map $(s, x) \mapsto \gamma_{x}(s)$ is locally a Lipschitz homeomorphism, it will follow that $\int_{\mathbb{R}^{n}} \rho \geq C_{3}>0$ for any admissible $\rho$, i.e. $\operatorname{Mod} A \geq C_{3}$ : this will establish the claim. 
Step 0 : Preliminary considerations. Let $\varepsilon>0$ be fixed and sufficiently small, in a sense we will specify later. We claim that there exist

$$
0=s_{0}<s_{1}<\cdots<s_{q}<s_{q+1}=T
$$

and neighbourhoods $U_{i} \subset \mathbb{R}^{n}$ of $\gamma\left(\left[s_{i}, s_{i+1}\right]\right), i=0, \ldots, q$, with the following properties:

- there exists a horizontal vector field $Z_{i} \in C^{\infty}\left(U_{i}, \mathbb{R}^{n}\right)$ such that

$$
\begin{aligned}
& Z_{i}(\gamma(s))=\dot{\gamma}(s) \text { for any } s \in\left[s_{i}, s_{i+1}\right] \text { and } \\
& 0<1-\varepsilon \leq\left|Z_{i}\right|_{\mathcal{X}} \leq 1+\varepsilon \text { on } U_{i}
\end{aligned}
$$

(recall that $|v| \mathcal{X}$ is the norm defined in (40));

- there is a $C^{\infty}$ change of coordinates $\left(u_{1}, \ldots, u_{n}\right)$ according to which $U_{i}$ is an open connected subset of $\mathbb{R}_{u_{1}, \ldots, u_{n}}^{n}$ with $\gamma\left(s_{i}\right)=0$ and $Z_{i}=\partial_{u_{1}}$.

We stress in particular that, according to such coordinates, we have

$$
\gamma(s)=\left(s-s_{i}, 0, \ldots, 0\right) \quad \text { for any } s \in\left[s_{i}, s_{i+1}\right] .
$$

The curves $\gamma_{x}$ will be constructed as a concatenation of integral lines of (multiples of) the vector fields $Z_{i}$ (see Steps $1,2,3$ ). We also point out that we do not know a priori that $\gamma$ is simple; otherwise one could easily prove our claim with $q=0$, which would make the construction of the curves $\gamma_{x}$ much easier.

Let us prove our claim. Since $\gamma$ is smooth and locally injective, for any $t \in[0, T]$ we can find

- $t^{\prime}=t^{\prime}(t), t^{\prime \prime}=t^{\prime \prime}(t)$ such that $t^{\prime}<t^{\prime \prime}$ and $0 \leq t^{\prime} \leq t \leq t^{\prime \prime} \leq T$,

- a neighbourhood $U_{t}$ of $\gamma(t)$

with the following properties:

- $\gamma_{\left[\left[t^{\prime}, t^{\prime \prime}\right]\right.}$ is simple and $\gamma\left(\left[t^{\prime}, t^{\prime \prime}\right]\right) \subset U_{t}$;

- there exists a horizontal vector field $Z_{t} \in C^{\infty}\left(U_{t}, \mathbb{R}^{n}\right)$ extending the horizontal $C^{\infty}$ vector $\dot{\gamma}$, that is, $Z_{t}(\gamma(s))=\dot{\gamma}(s)$ for any $s \in\left[t^{\prime}, t^{\prime \prime}\right]$;

- there exists $\varepsilon>0$ such that $1-\varepsilon<\left|Z_{t}\right| \mathcal{X}<1+\varepsilon$ on $U_{t}$ (since $|\dot{\gamma}|=1$, i.e. $\left|Z_{t}\right|_{\mathcal{X}}=1$ on $\gamma\left(\left[t^{\prime}, t^{\prime \prime}\right]\right)$, it will be enough to take $U_{t}$ sufficiently small);

- there is a $C^{\infty}$ change of coordinates according to which $U_{t}$ is an open connected subset of $\mathbb{R}_{u_{1}, \ldots, u_{n}}^{n}$ with $\gamma\left(t^{\prime}\right)=0$ and $Z_{t}=\partial_{u_{1}}$ (this follows from the Rectifiability Theorem for ODEs; see e.g. [1]).

In particular, according to such coordinates, we have

$$
\gamma(s)=\left(s-t^{\prime}, 0, \ldots, 0\right) \quad \text { for any } s \in\left[t^{\prime}, t^{\prime \prime}\right] .
$$

By compactness of $[0, T]$ and the fact that none of the intervals $\left[t^{\prime}, t^{\prime \prime}\right]$ has an empty interior, there exists a finite set $\left\{t_{i}\right\}_{i=0}^{q} \subset[0, T]$ such that

$$
[0, T] \subset \bigcup_{i=0}^{q}\left[t_{i}^{\prime}, t_{i}^{\prime \prime}\right], \quad \text { where } t_{i}^{\prime}:=t^{\prime}\left(t_{i}\right), t_{i}^{\prime \prime}:=t^{\prime \prime}\left(t_{i}\right) .
$$

We may assume that the covering $\left\{\left[t_{i}^{\prime}, t_{i}^{\prime \prime}\right]\right\}_{i}$ is minimal, in the sense that there exists no couple $i, j$ with $i \neq j$ and such that $\left[t_{i}^{\prime}, t_{i}^{\prime \prime}\right] \subset\left[t_{j}^{\prime}, t_{j}^{\prime \prime}\right]$. In particular $t_{i}^{\prime} \neq t_{j}^{\prime}$, $t_{i}^{\prime \prime} \neq t_{j}^{\prime \prime}$ whenever $i \neq j$; moreover, the implication

$$
t_{i}^{\prime}<t_{j}^{\prime} \Longrightarrow t_{i}^{\prime \prime}<t_{j}^{\prime \prime}
$$

holds. Let our intervals be ordered so that $i<j \Rightarrow t_{i}^{\prime}<t_{j}^{\prime}$. One must have

$$
t_{i+1}^{\prime} \leq t_{i}^{\prime \prime} \quad \text { for any } i
$$


otherwise,

$$
\left(t_{i}^{\prime \prime}, t_{i+1}^{\prime}\right) \cap\left[t_{j}^{\prime}, t_{j}^{\prime \prime}\right]=\emptyset \text { for any } j=0, \ldots, q
$$

(it is sufficient to consider separately the cases $j \leq i$ and $j>i$ and to use (12)). This would lead to a contradiction since $\left(t_{i}^{\prime \prime}, t_{i+1}^{\prime}\right) \not \subset \bigcup_{j}\left[t_{j}^{\prime}, t_{j}^{\prime \prime}\right]$.

Let us set $s_{i}:=t_{i}^{\prime}, i=0, \ldots, q$ and $s_{q+1}:=T=t_{q}^{\prime \prime}$; we have by (13),

$$
\left[s_{i}, s_{i+1}\right]=\left[t_{i}^{\prime}, t_{i+1}^{\prime}\right] \subset\left[t_{i}^{\prime}, t_{i}^{\prime \prime}\right]
$$

and our claim follows by setting $U_{i}:=U_{t_{i}}$ and $Z_{i}:=Z_{t_{i}}$.

Step 1 : Construction of the horizontal curves $\gamma_{x}$ on $\left[0, s_{1}\right]$. Let us consider $\gamma_{\mid\left[0, s_{1}\right]}$ in the coordinate chart $\left(u_{1}, \ldots, u_{n}\right)$ for $U_{0}$ given by Step 0: such a curve appears as the path $s \mapsto(s, 0, \ldots, 0)$; moreover, $\gamma(0)=0$ and $Z_{0}=\partial_{u_{1}}$.

We can fix an open set $\Sigma_{0} \subset\left\{u_{1}=0\right\} \equiv \mathbb{R}^{n-1}$ with $\gamma(0)=0 \in \Sigma_{0}$; we may assume that $\left[0, s_{1}\right] \times \Sigma_{0} \Subset U_{0}$. Therefore the map

$$
\gamma_{x}(s):=\exp \left(s Z_{0}\right)(x)=(s, x) \in \mathbb{R} \times \mathbb{R}^{n-1}
$$

is well defined for $s \in\left[0, s_{1}\right], x \in \Sigma_{0}$ and, since $\dot{\gamma}_{x}=\partial_{u_{1}}=Z_{0}$, it is a horizontal curve in $U_{0}$ with $1-\varepsilon<\left|\dot{\gamma}_{x}\right|_{\mathcal{X}}<1+\varepsilon$. Define

$$
\Sigma_{1}:=\left\{s_{1}\right\} \times \Sigma_{0}=\bigcup_{x \in \Sigma_{0}} \gamma_{x}\left(s_{1}\right) .
$$

Step 2 : Extension of $\gamma_{x}$ to $\left[s_{1}, s_{2}\right]$. Possibly restricting $\Sigma_{0}$, the $C^{\infty}$ hypersurface $\Sigma_{1}$ is contained in $U_{1} \cdot \Sigma_{1}$ is a transversal to $Z_{1}=\partial_{u_{1}}$ at $\gamma\left(s_{1}\right)=0 \in \Sigma_{1}$ (where $u_{1}, \ldots, u_{n}$ are now the coordinates chosen for $U_{1}$ ). Possibly considering a smaller $\Sigma_{0}, \Sigma_{1}$ is then a $C^{\infty}$ graph over the hyperplane $\left\{u_{1}=0\right\}$, i.e.

$$
\Sigma_{1}=\left\{\left(\phi_{1}(y), y\right): y \in V_{1}\right\}
$$

for some $C^{\infty}$ real map $\phi_{1}$ defined on an open subset $V_{1} \subset\left\{u_{1}=0\right\}$.

We have $\phi_{1}(0)=0$ and, after restricting $\Sigma_{0}$, we may also assume $\left|\phi_{1}\right|<\delta$, for some $\delta$ to be chosen later.

We are going to extend the curves $\left\{\gamma_{x}\right\}_{x \in \Sigma_{0}}$ to $\left[0, s_{2}\right]$. We have $\gamma_{x}\left(s_{1}\right)=$ $\left(\phi_{1}\left(v_{x}^{1}\right), v_{x}^{1}\right) \in \Sigma_{1}$ for some $v_{x}^{1} \in V_{1}$. We set

$$
\gamma_{x}(s):=\left(\phi_{1}\left(v_{x}^{1}\right)+\frac{s_{2}-s_{1}-\phi_{1}\left(v_{x}^{1}\right)}{s_{2}-s_{1}}\left(s-s_{1}\right), v_{x}^{1}\right), \quad s \in\left[s_{1}, s_{2}\right] .
$$

In other words, $\gamma_{x \mid\left[s_{1}, s_{2}\right]}$ is a parametrization of the segment joining $\left(\phi\left(v_{x}^{1}\right), v_{x}^{1}\right)$ and $\left(s_{2}-s_{1}, v_{x}^{1}\right)$; moreover, it is a horizontal curve because its derivative is a multiple of $\partial_{u_{1}}=Z_{1}$. It is clear that, possibly restricting $\Sigma_{0}, \gamma_{x}(s)$ is well defined for any $x \in \Sigma_{0}, s \in\left[0, s_{2}\right]$ and is a horizontal curve in $U_{1}$. Set

$$
\Sigma_{2}:=\left\{s_{2}-s_{1}\right\} \times V_{1}=\bigcup_{x \in \Sigma_{0}} \gamma_{x}\left(s_{2}\right)
$$

to be the $C^{\infty}$ surface consisting of the endpoints $\gamma_{x}\left(s_{2}\right)$ : in the chart for $U_{1}, \Sigma_{2}$ appears as a portion of a hyperplane.

Step 3 : Extension of $\gamma_{x}$ to the whole $[0, T]$. As in Step 2 one can prove that, in the coordinate chart for $U_{2}$ given by Step 0 , the surface $\Sigma_{2}$ is the graph of a $C^{\infty}$ map 


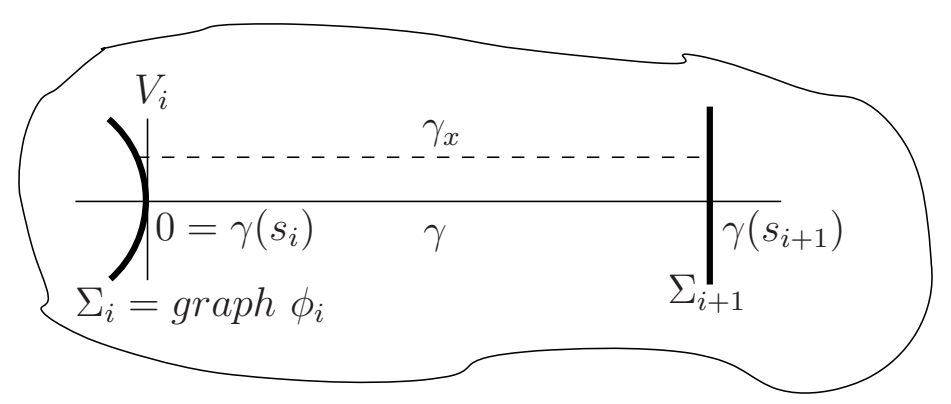

Figure 1. Construction of $\gamma_{x}$ in the chart $\left(U_{i}, \psi_{i}\right)$

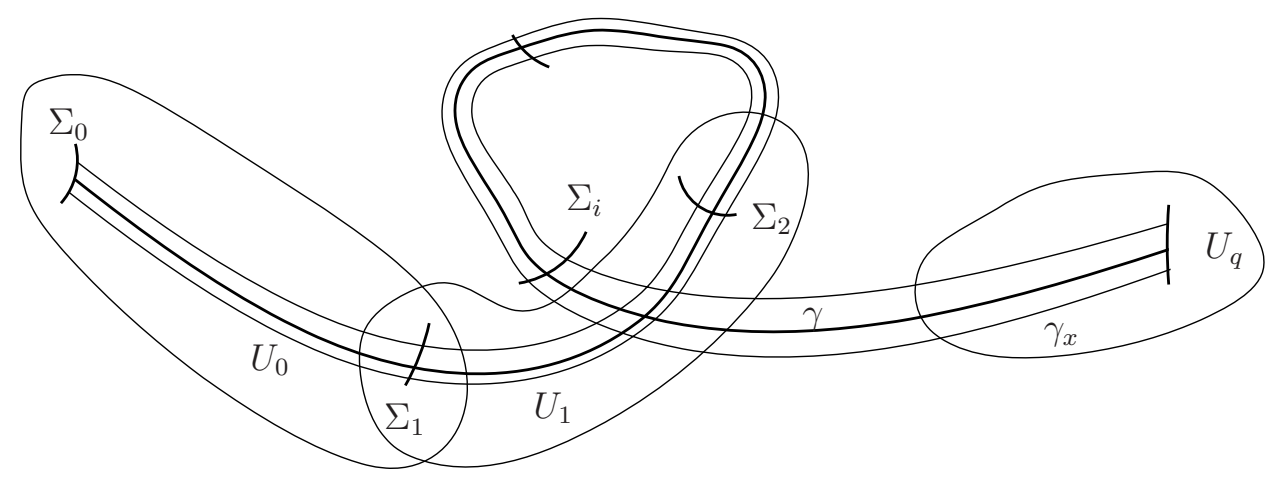

Figure 2. The curves $\gamma_{x}$ span a tubular neighbourhood of $\gamma$

$\phi_{2}: V_{2} \rightarrow \mathbb{R}$ with $\left|\phi_{2}\right|<\delta$; in doing so, it could be necessary to reduce $\Sigma_{0}$ and/or $\delta$. We have $\gamma_{x}\left(s_{2}\right)=\left(\phi_{2}\left(v_{x}^{2}\right), v_{x}^{2}\right)$ for some $v_{x}^{2} \in V_{2}$. We extend $\gamma_{x}$ by

$$
\gamma_{x}(s):=\left(\phi_{2}\left(v_{x}^{2}\right)+\frac{s_{3}-s_{2}-\phi_{2}\left(v_{x}^{2}\right)}{s_{3}-s_{2}}\left(s-s_{2}\right), v_{x}^{2}\right), \quad s \in\left[s_{2}, s_{3}\right]
$$

and set $\Sigma_{3}:=\bigcup_{x \in \Sigma_{0}} \gamma_{x}\left(s_{3}\right)=\left\{s_{3}-s_{2}\right\} \times V_{2}$.

By induction on $i$ we end up with a horizontal curve $\gamma_{x}:[0, T] \rightarrow \mathbb{R}^{n}$ defined for $x \in \Sigma_{0}$. The endpoints of each $\gamma_{x}$ are, respectively, a point of $\Sigma_{0}$ and a point lying on a $C^{\infty}$ surface $\Sigma_{q+1} \subset U_{q}$.

Step $4:$ Each $\gamma_{x}$ belongs to $\Gamma$. Again restricting $\Sigma_{0}$, we may assume that $\Sigma_{0} \subset B_{1}$ and $\Sigma_{q+1} \subset B_{2}$, so that

$$
\gamma_{x}(0) \in B_{1} \text { and } \gamma_{x}(T) \in B_{2} \text { for any } x \in \Sigma_{0} .
$$

If $\delta$ is sufficiently small, for any $x \in \Sigma_{0}$ and $s \in\left[s_{i}, s_{i+1}\right]$ there exists $v_{x} \in V_{i}$ such that

$$
\left|\dot{\gamma}_{x}(s)\right|_{\mathcal{X}}=\left|\frac{s_{i+1}-s_{i}-\phi_{i}\left(v_{x}\right)}{s_{i+1}-s_{i}}\right|\left|\partial_{u_{1}^{i}}\right|_{\mathcal{X}} \leq 1+2 \varepsilon
$$


because, by (11), $\left|\partial_{u_{1}^{i}}\right| \mathcal{X}=\left|Z_{i}\right| \leq 1+\varepsilon$; here, $u_{1}^{i}, \ldots, u_{n}^{i}$ denote the coordinates for $U_{i}$ given by Step 0 . Therefore

$$
l\left(\gamma_{x}\right) \leq(1+2 \varepsilon) T \leq(1+2 \varepsilon)\left(d\left(B_{1}, B_{2}\right)+\frac{\tau}{2}\right)
$$

and then $l\left(\gamma_{x}\right) \leq d\left(B_{1}, B_{2}\right)+\tau$ provided $\varepsilon$ is sufficiently small (depending only on $d\left(B_{1}, B_{2}\right)$ and $\left.\tau\right)$. In particular, $\gamma_{x} \in \Gamma$ (with $\left.C=1\right)$.

Step 5 : The Jacobian of the map $(s, x) \mapsto \gamma_{x}(s)$. By construction, the Lipschitz map

$$
[0, T] \times \Sigma_{0} \ni(s, x) \stackrel{F}{\longmapsto} \gamma_{x}(s) \in \mathbb{R}^{n}
$$

is locally a diffeomorphism; it is not necessarily injective because, for example, $\gamma$ could have self-intersections. Nevertheless, for any $i$, the map $F_{\left[\left[s_{i}, s_{i+1}\right] \times \Sigma_{0}\right.}$ is a diffeomorphism (onto its image). Possibly restricting $\Sigma_{0}$ we can suppose that

$$
C:=\inf \left\{|\operatorname{det} J F(s, x)|:(s, x) \in[0, T] \times \Sigma_{0}\right\}>0 .
$$

Step 6 : Conclusion. Let $\rho$ be a positive Borel function on $\mathbb{R}^{n}$ with $\int_{c} \rho \geq 1$ for any $c \in \Gamma$. Since $\int_{\gamma_{x}} \rho \geq 1$ for any $x \in \Sigma_{0}$, there exists a Borel map $I: \Sigma_{0} \rightarrow\{0, \ldots, q\}$ such that

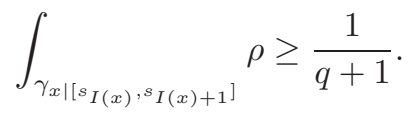

In particular there exists $j \in\{0,1, \ldots, q\}$ such that

$$
\mathcal{L}^{n-1}\left(\left\{x \in \Sigma_{0}: I(x)=j\right\}\right) \geq \frac{1}{q+1} \mathcal{L}^{n-1}\left(\Sigma_{0}\right) .
$$

We have therefore

$$
\begin{aligned}
\int_{\mathbb{R}^{n}} \rho d \mu & \geq \int_{F\left(\left[s_{j}, s_{j+1}\right] \times \Sigma_{0}\right)} \rho d \mu \\
& =\int_{\left[s_{j}, s_{j+1}\right] \times \Sigma_{0}}|\operatorname{det} J F(s, x)| \rho(F(s, x)) d \mathcal{L}^{n}(s, x),
\end{aligned}
$$

where we have used a change of variable. It follows that

$$
\begin{aligned}
\int_{\mathbb{R}^{n}} \rho d \mu & \geq C \int_{\Sigma_{0}} \int_{s_{j}}^{s_{j+1}} \rho\left(\gamma_{x}(s)\right) d s d x \\
& \geq \frac{C}{1+2 \varepsilon} \int_{\Sigma_{0}} \int_{s_{j}}^{s_{j+1}} \rho\left(\gamma_{x}(s)\right)\left|\dot{\gamma}_{x}(s)\right|_{\mathcal{X}} d s d x \\
& \geq \frac{C}{1+2 \varepsilon} \int_{\left\{x \in \Sigma_{0}: I(x)=j\right\}}\left(\int_{\gamma_{x}} \rho\right) d x \\
& \geq \frac{C}{1+2 \varepsilon} \cdot \frac{1}{q+1} \cdot \mathcal{L}^{n-1}\left(\Sigma_{0}\right) \cdot \frac{1}{q+1}>0
\end{aligned}
$$

and then $\operatorname{Mod} A>0$ with $A=\left\{\gamma_{x}\right\}_{x \in \Sigma_{0}}$, as claimed.

Therefore, by Theorems 2.9 and the weak Fubini property, we can conclude the following equivalence.

Corollary 3.5. In Riemannian and sub-Riemannian manifolds, endowed with their natural volume measure, AMLEs and SAMLEs are equivalent notions.

We also state the following corollary, which will be of use later. 
Corollary 3.6. Let $\wp=\wp(x)$ be a property holding for $\mathcal{L}^{n}$-a.e. $x \in \mathbb{R}^{n}$ and let $x_{0}, y_{0} \in \mathbb{R}^{n}$ be fixed. Then, for any $\varepsilon>0$ there exist two points $x_{\varepsilon} \in B_{\varepsilon}\left(x_{0}\right), y_{\varepsilon} \in$ $B_{\varepsilon}\left(y_{0}\right)$ and a horizontal curve $\gamma:[0, T] \rightarrow \mathbb{R}^{n}$ with $\gamma(0)=x_{\varepsilon}$ and $\gamma(T)=y_{\varepsilon}$ such that $l(\gamma)<d\left(x_{0}, y_{0}\right)+\varepsilon$ and $\wp$ holds at $\gamma(t)$ for $\mathcal{L}^{1}$-a.e. $t \in[0, T]$.

Proof. Consider $B_{1}:=B_{\varepsilon}\left(x_{0}\right), B_{2}:=B_{\varepsilon}\left(y_{0}\right)$. By repeating the construction given in Theorem 3.4 (with $\tau:=\varepsilon$ ) we can find $T>0$ and

- a $C^{\infty}$ hypersurface $\Sigma_{0} \subset B_{\varepsilon}\left(x_{0}\right)$;

- $0=s_{0}<s_{1}<\cdots<s_{q}<s_{q+1}=T$;

- a Lipschitz continuous map $F:[0, T] \times \Sigma_{0} \rightarrow \mathbb{R}^{n}$

such that

- for any $i, F$ is a $C^{\infty}$ diffeomorphism from $\left(\left[s_{i}, s_{i+1}\right] \times \Sigma_{0}\right)$ onto its image;

- for any $x \in \Sigma_{0}$, the curve $\gamma_{x}:=F(\cdot, x)$ is horizontal, $\gamma_{x}(0) \in B_{\varepsilon}\left(x_{0}\right)$ and $\gamma_{x}(T) \in B_{\varepsilon}\left(y_{0}\right)$

- the length of each $\gamma_{x}$ is less than $d\left(x_{0}, y_{0}\right)+\varepsilon$.

Let $\left(U_{i}, \psi_{i}\right)$ be the charts utilized in the same construction and recall that, for any $x \in \Sigma_{0}$ and any $i$ the curve $\gamma_{x \mid\left[s_{i}, s_{i+1}\right]}$ is a straight horizontal segment according to the coordinates $\left(U_{i}, \psi_{i}\right)$. By the classical Fubini theorem, for $\mathcal{H}^{n-1}$-a.e. $x \in \Sigma_{0}$ the property $\wp$ holds at $\gamma_{x}(t)$ for $\mathcal{L}^{1}$-a.e. $t \in\left[s_{i}, s_{i+1}\right]$. As a consequence, for $\mathcal{H}^{n-1}$-a.e. $x \in \Sigma_{0}$ the property $\wp$ holds at $\gamma_{x}(t)$ for $\mathcal{L}^{1}$-a.e. $t \in[0, T]$, and this concludes the proof.

Remark 3.7. The proof of Corollary 3.6 shows that there are plenty of horizontal curves $\gamma$ as in the statement.

\section{Equivalence Between abSOlutely minimizing LipsChitZ EXTENSIONS AND INFINITY HARMONIC FUNCTIONS}

As we have already pointed out in the introduction, it is known that, in Euclidean spaces, AMLEs and infinity harmonic functions are equivalent notions. We are going to introduce the corresponding horizontal operators and to investigate the same relation in Riemannian and sub-Riemannian manifolds. As usual, $\mathcal{X}=\left\{X_{1}(x), \ldots, X_{m}(x)\right\}$ denotes a family of smooth vector fields in $\mathbb{R}^{n}$ satisfying the Hörmander condition and $d$ is the associated Carnot-Carathéodory distance.

Definition 4.1. We define the horizontal gradient of $u: \mathbb{R}^{n} \rightarrow \mathbb{R}$ as

$$
D_{\mathcal{X}} u:=\left(X_{1} u, \ldots, X_{m} u\right)
$$

and the symmetrized matrix of horizontal second-order derivatives of $u$ as

$$
\left(D_{\mathcal{X}}^{2} u\right)_{i, j}^{\star}:=\frac{1}{2}\left(X_{i} X_{j} u+X_{j} X_{i} u\right), \text { for } i, j=1, \ldots, m .
$$

Using the previous intrinsic differential operators, we introduce the (renormalized) infinity Laplace operator by

$$
\Delta_{\infty, \mathcal{X}} u:=\left\langle\left(D_{\mathcal{X}}^{2} u\right)^{\star} D_{\mathcal{X}} u, D_{\mathcal{X}} u\right\rangle .
$$

The equation under consideration is the infinity Laplace equation given by

$$
\Delta_{\infty, \mathcal{X}} u=0 .
$$

We say that a function $u$ is of class $C_{\mathcal{X}}^{1}$ if $u$ is continuous and $X_{i} u$ is continuous for $i=1, \ldots, m$. Analogously, $u \in C_{\mathcal{X}}^{k}$ with $k \geq 1$ if $u$ is continuous with its first $k$ 
horizontal derivatives. Of course, we do not specify if such continuity is w.r.t. the metric $d$ or the usual Euclidean one because, under the Hörmander condition, the topology induced by $d$ is equivalent to the Euclidean one.

Definition 4.2. We call a (horizontal) infinity harmonic function any viscosity solution of (14); that is, an infinity harmonic function $u$ is a continuous function such that

(1) if $\phi \in C_{\mathcal{X}}^{2}$ is such that $u-\phi$ has a local maximum at $x_{0}$, then $-\Delta_{\infty, \mathcal{X}} \phi\left(x_{0}\right)$ $\leq 0$ (subsolution property);

(2) if $\phi \in C_{\mathcal{X}}^{2}$ is such that $u-\phi$ has a local minimum at $x_{0}$, then $-\Delta_{\infty, \mathcal{X}} \psi\left(x_{0}\right) \geq$ 0 (supersolution property).

Our aim is that of understanding whether, given a bounded domain $\Omega \subset \mathbb{R}^{n}$ and $g \in \operatorname{Lip}(\partial \Omega, d)$, a function $u$ is the AMLE of $g$ on $\bar{\Omega}$ if and only if it is infinity harmonic on $\Omega$ with $u=g$ on $\partial \Omega$. In order to obtain this equivalence, we first need to introduce the concept of an absolutely gradient minimizing extension. In fact, it is well known that, at least in the Euclidean case, the infinity Laplacian is the Aronsson equation of the $L^{\infty}$-norm of the gradient; in particular, infinity harmonic functions are those functions minimizing such a norm.

As is common in the literature, we hereafter denote by $W_{\mathcal{X}}^{1, \infty}(\Omega)$ the space of real functions on $\Omega$ whose first-order horizontal derivatives are essentially bounded.

Definition 4.3. Let $\Omega \subset \mathbb{R}^{n}$ be a bounded domain and $g \in \operatorname{Lip}(\partial \Omega, d)$. We say that $u \in W_{\mathcal{X}}^{1, \infty}(\Omega)$ is an absolutely gradient minimizing extension (AGME) of $g$ on $\bar{\Omega}$ if

(1) $u=g$ on $\partial \Omega$;

(2) for any $U \Subset \Omega$ open, if $v \in W_{0}^{1, \infty}(U)$ with $v=u$ on $\partial U$, then

$$
\left\|D_{\mathcal{X}} u\right\|_{\infty, U} \leq\left\|D_{\mathcal{X}} v\right\|_{\infty, U} .
$$

The following result was proved by C. Wang [25].

Theorem 4.4. If $u$ is AGME in a Carnot-Carathéodory space, then $u$ is a viscosity solution of (14).

Still in [25], the author also proved that, in any Carnot group, the viscosity solutions of

$$
\left\{\begin{aligned}
\Delta_{\infty, \mathcal{X}} u & =0, & & \text { on } \Omega \\
u & =g, & & \text { on } \partial \Omega
\end{aligned}\right.
$$

are unique. The previous uniqueness result is also known in Riemannian manifolds ([3]) and in Grušin-type spaces ([5]). Uniqueness for the problem (15) implies that the reverse implication (i.e. that infinity harmonic functions are AGMEs) holds, thus establishing the full equivalence between AGMEs and infinity harmonic functions.

We can summarize the previous results in the following corollary.

Corollary 4.5 ([25], 3], 5]). In Riemanian manifolds, Carnot groups and Grušintype spaces, a function is AGME if and only if it is infinity harmonic.

Unfortunately, in general Carnot-Carathéodory spaces the uniqueness for problem (15), as well as the implication "infinity harmonic $\Rightarrow$ AGME", are still open 
problems. Therefore the previous full equivalence is not yet established in the general setting.

We also want to study the relationships between the notions of AMLE and AGME. In the Euclidean setting it is known that $\operatorname{Lip}(u, U)$ equals $\|D u\|_{L^{\infty}(U)}$ for any Lipschitz function $u$ on a convex set $U \subset \mathbb{R}^{n}$. If $U$ is not convex only one inequality holds, namely $\operatorname{Lip}(u, U) \geq\|D u\|_{L^{\infty}(U)}$. The reverse inequality is in general false, as the following example shows.

Example 4.6. Define $\Omega=B_{1}(0)=\left\{(x, y) \in \mathbb{R}^{2} \mid x^{2}+y^{2}<1\right\}$ and let $\overline{B_{r}}(0)=$ $\left\{(x, y) \in \mathbb{R}^{2} \mid x^{2}+y^{2} \leq r^{2}\right\}$, with $0<r \ll 1$ fixed. Consider the open set

$$
\widetilde{\Omega}=\Omega \backslash\left(\overline{B_{r}}(0) \cup\left\{(x, y) \in \mathbb{R}^{2} \mid y=0, x \leq 0\right\}\right) .
$$

Then $\widetilde{\Omega}$ is connected and bounded but not convex. Let us consider the "angle" function $u: \widetilde{\Omega} \rightarrow \mathbb{R}$ defined by

$$
u(x, y)= \begin{cases}\arctan \frac{y}{x} & \text { if } x>0 \\ \pi+\arctan \frac{y}{x} & \text { if } x<0 \text { and } y>0 \\ -\pi+\arctan \frac{y}{x} & \text { if } x<0 \text { and } y<0 \\ \pi / 2 & \text { if } x=0 \text { and } y>r \\ -\pi / 2 & \text { if } x=0 \text { and } y<-r .\end{cases}
$$

It is easy to see that

$$
|D u|=\frac{1}{\sqrt{x^{2}+y^{2}}}<\frac{1}{r}, \quad \text { for any }(x, y) \in \widetilde{\Omega},
$$

so that $\|D u\|_{\infty, \widetilde{\Omega}} \leq \frac{1}{r}$.

Now look at points $P=\left(x_{P}, y_{P}\right), Q=\left(x_{Q}, y_{Q}\right) \in \widetilde{\Omega}$ such that $x_{P}=x_{Q} \approx-1$ and $0<y_{P} \ll 1$ and $-1 \ll y_{Q}<0$, so that $d(P, Q)<\varepsilon \ll 1$.

We have

$$
u(P) \approx \pi \quad \text { while } \quad u(Q) \approx-\pi
$$

Hence

$$
\frac{|u(P)-u(Q)|}{|P-Q|} \approx \frac{2 \pi}{\varepsilon}
$$

and then $\operatorname{Lip}(u, \widetilde{\Omega})=+\infty$, whence $\operatorname{Lip}(u, \widetilde{\Omega})>\|D u\|_{\infty, \widetilde{\Omega}}$.

An analogous result holds for geodesically convex sets in Carnot-Carathéodory spaces; in this setting, however, the geometry of geodesically convex sets is not as well-behaved as in Euclidean spaces. For example, in the Heisenberg group the only geodesically convex sets are the whole space, the empty set and the geodesics themselves 18.

However, let us note that balls are starshaped (i.e. geodesically convex with respect to an interior fixed point) w.r.t. the center in any length space.

Next, we show that in any Carnot-Carathéodory space the local Lipschitz norm introduced in (9) and the $L^{\infty}$-norm of the gradient are indeed the same object.

Theorem 4.7. Let $\left(\mathbb{R}^{n}, \mathcal{X},\langle,\rangle_{g}\right)$ be a Riemannian or sub-Riemannian manifold and $d(x, y)$ the associated Riemannian or, respectively, Carnot-Carathéodory distance. Let $U \subset \mathbb{R}^{n}$ be an open set and $u: U \rightarrow \mathbb{R}$ be a Lipschitz function. Then

$$
\widehat{\operatorname{Lip}}(u, U)=\left\|D_{\mathcal{X}} u\right\|_{\infty, U} \text {. }
$$


Proof. In [10] (see also [11]) it was proved that, under the Hörmander condition, for any open and bounded set $\Omega \subset U$ and for any Lipschitz function $u: U \rightarrow \mathbb{R}$, we have

$$
\left\|D_{\mathcal{X}} u\right\|_{\infty, \Omega} \leq \operatorname{Lip}(u, \Omega) .
$$

Using this result in $\Omega=B_{R}\left(x_{0}\right) \subset U$ and passing to the limit as $R \rightarrow 0^{+}$we find

$$
\left|D_{\mathcal{X}} u(x)\right| \leq \operatorname{Lip} u(x), \quad \text { a.e. } x \in U \text {. }
$$

On considering the essential supremum we achieve

$$
\left\|D_{\mathcal{X}} u\right\|_{\infty, U} \leq \underset{x \in U}{\operatorname{ess} \sup } \operatorname{Lip} u(x)=\widehat{\operatorname{Lip}}(u, U) .
$$

We are left to prove the reverse inequality.

Let $x \in U$ be fixed and $R>0$ be such that $B_{R}(x) \subset U$ and fix $y \in B_{R}(x)$. By Corollary [3.6, for any $\varepsilon>0$ there exists a horizontal curve $\gamma_{\varepsilon}:[0, T] \rightarrow \mathbb{R}^{n}$ such that $\gamma_{\varepsilon}(0)=x_{\varepsilon}, \gamma_{\varepsilon}(T)=y_{\varepsilon}$ and

$$
d\left(x, x_{\varepsilon}\right)<\varepsilon, d\left(y, y_{\varepsilon}\right)<\varepsilon, l\left(\gamma_{\varepsilon}\right)<d(x, y)+3 \varepsilon,
$$

$u$ is horizontally differentiable at $\gamma_{\varepsilon}(t)$ for $\mathcal{L}^{1}$-a.e. $t \in[0, T]$.

If $\varepsilon$ is such that $d(x, y)+3 \varepsilon<R$, then $\operatorname{Im} \gamma_{\varepsilon} \subset B_{R}(x) \subset U$ and, again by Corollary 3.6. we may assume that

$$
\left|D_{\mathcal{X}} u\left(\gamma_{\varepsilon}(t)\right)\right| \leq\left\|D_{\mathcal{X}} u\right\|_{\infty, B_{R}(x)} \text { for } \mathcal{L}^{1} \text {-a.e. } t \in[0, T] .
$$

Therefore

$$
\begin{aligned}
\left|u\left(x_{\varepsilon}\right)-u\left(y_{\varepsilon}\right)\right| & \leq\left|\int_{0}^{T} \frac{d}{d t} u\left(\gamma_{\varepsilon}(t)\right) d t\right|=\left|\int_{0}^{T} D_{\mathcal{X}} u\left(\gamma_{\varepsilon}(t)\right) \cdot \dot{\gamma}_{\varepsilon}(t) d t\right| \\
& \leq\left\|D_{\mathcal{X}} u\right\|_{\infty, B_{R}(x)} \int_{0}^{T}\left|\dot{\gamma}_{\varepsilon}(t)\right| d t=\left\|D_{\mathcal{X}} u\right\|_{\infty, B_{R}(x)} l\left(\gamma_{\varepsilon}\right) \\
& =\left\|D_{\mathcal{X}} u\right\|_{\infty, B_{R}(x)}(d(x, y)+3 \varepsilon) .
\end{aligned}
$$

Taking into account the continuity of $u$, as $\varepsilon \rightarrow 0$ we obtain

$$
|u(x)-u(y)| \leq\left\|D_{\mathcal{X}} u\right\|_{\infty, B_{R}(x)} d(x, y) .
$$

Taking the supremum among $y \in B_{R}(x) \backslash\{x\}$ and passing to the limit as $R \rightarrow 0^{+}$, we can conclude

i.e.

$$
\operatorname{Lip} u(x) \leq\left\|D_{\mathcal{X}} u\right\|_{\infty, U}, \quad \forall x \in U,
$$

$$
\widehat{\operatorname{Lip}}(u, U)=\underset{x \in U}{\operatorname{ess} \sup } \operatorname{Lip} u(x) \leq\left\|D_{\mathcal{X}} u\right\|_{\infty, U},
$$

which concludes the proof.

In the particular case of a Carnot group, Taylor approximation allows us to prove the identity between Lip $u(x)$ and $\left|D_{\mathcal{X}} u(x)\right|$ for a.e. $x$. This is not necessary in order to study the relation between AMLEs and infinity harmonic functions; nevertheless, we decided to include it since it can be useful in different contexts.

The intrinsic Taylor's approximation in Carnot groups was proved in 21; more precisely, if $u$ is a Lipschitz function on a Carnot group $\mathbb{G}$, then $u$ is horizontally differentiable at a.e. $x \in \mathbb{G}$, i.e.

$$
u(y \cdot x)=u(x)+\left\langle D_{\mathcal{X}} u(x), \bar{y}\right\rangle+o\left(\|y\|_{C C}\right),
$$

where $\bar{y}$ denotes the horizontal projection of $y$ onto the horizontal layer. 
Proposition 4.8. Let $\mathbb{G}$ be a Carnot group, $u: \mathbb{G} \rightarrow \mathbb{R}$ a Lipschitz function and $x \in \mathbb{G}$ a point such that $u$ is horizontally differentiable at $x$. Then

$$
\operatorname{Lip} u(x)=\left|D_{\mathcal{X}} u(x)\right| \text {. }
$$

Proof. Let $R>0$ be fixed and let $h$ be a "horizontal" point (i.e., $\bar{h}=h$ ), to be chosen later, such that $\|h\|_{C C}=1$. Let $s \in(0, R)$ and define $y:=x \cdot \delta_{s}(h) \in B_{R}(x)$. By (16) we have

$$
\begin{aligned}
u(y) & =u(x)+\left\langle D_{\mathcal{X}} u(x), \overline{x^{-1} \cdot y}\right\rangle+o\left(\left\|x^{-1} \cdot y\right\|_{C C}\right) \\
& =\left\langle D_{\mathcal{X}} u(x), \overline{\delta_{s}(h)}\right\rangle+o\left(\left\|\delta_{s}(h)\right\|_{C C}\right)
\end{aligned}
$$

so that

$$
u(y)-u(x)=s\left\langle D_{\mathcal{X}} u(x), h\right\rangle+o(s) .
$$

Since $d(x, y)=\left\|\delta_{s}(h)\right\|_{C C}=s$ we get

$$
\frac{u(y)-u(x)}{d(x, y)}=\left\langle D_{\mathcal{X}} u(x), h\right\rangle+o(1) .
$$

After choosing $h:=\frac{D_{\mathcal{X}} u(x)}{\left|D_{\mathcal{X}} u(x)\right|}$, we obtain

$$
\sup _{y \in B_{R}(x) \backslash\left\{x_{0}\right\}} \frac{u(y)-u(x)}{d(x, y)} \geq\left|D_{\mathcal{X}} u(x)\right|+o(1) .
$$

On passing to the limit as $R \rightarrow 0^{+}$we conclude

$$
\text { Lip } u(x) \geq\left|D_{\mathcal{X}} u(x)\right| .
$$

In order to get the reverse inequality, we proceed in a similar way. We write the first-order Taylor's approximation at a point $y=x \cdot \delta_{s}(h)$ with $\|h\|_{C C}=1$ but $h$ not necessarily horizontal. We then have

$$
u(y)-u(x)=s\left\langle D_{\mathcal{X}} u(x), \bar{h}\right\rangle+o(s) .
$$

Using the Cauchy-Schwarz inequality, we find

$$
\frac{|u(y)-u(x)|}{d(y, x)} \leq\left|D_{\mathcal{X}} u(x)\right||\bar{h}|+o(1)
$$

Note that $|\bar{h}| \leq\|h\|_{C C}=1$ and so

$$
\frac{|u(y)-u(x)|}{d(y, x)} \leq\left|D_{\mathcal{X}} u(x)\right|+o(1)
$$

for any $y \in B_{R}(x)$. Taking the supremum and then passing to the limit as $R \rightarrow 0^{+}$, we can conclude

$$
\operatorname{Lip} u(x) \leq\left|D_{\mathcal{X}} u(x)\right| .
$$

Inequalities (18) and (19) are enough to conclude the proof of the theorem.

Remark 4.9. Recall that, whenever $u$ is Lipschitz w.r.t. the Carnot-Carathéodory distance in some bounded set $\Omega \subset \mathbb{R}^{n}$, then $D_{\mathcal{X}} u \in L^{\infty}(\Omega)$ (see [10] and 11]). In the particular case of a Carnot group the result is indeed stronger: the Lipschitz continuity w.r.t. the Carnot-Carathéodory distance implies that the function is also horizontally differentiable a.e. (Pansu-Rademacher Theorem, e.g. in [17]). 
Before stating the final result, i.e. the equivalence between AMLEs and infinity harmonic functions, we would like to summarize all the equivalences that have been obtained so far:

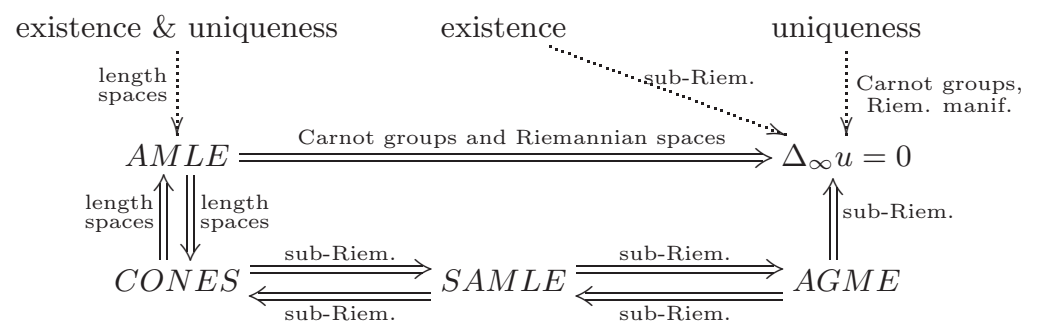

We can conclude the following.

Theorem 4.10. Let $\left(\mathbb{R}^{n}, \mathcal{X},\langle,\rangle_{g}\right)$ be a Riemannian or sub-Riemannian manifold, $\Omega \subset \mathbb{R}^{n}$ open and bounded and let $g: \partial \Omega \rightarrow \mathbb{R}$ be a Lipschitz boundary datum. If $u: \bar{\Omega} \rightarrow \mathbb{R}$ is the AMLE of $g$, then $u$ is infinity harmonic on $\Omega$.

Moreover, in the particular cases of Riemannian manifolds, Carnot groups and Grušin spaces, the reverse implication also holds.

Proof. By the weak Fubini property, we know that AMLEs and SAMLEs are equivalent notions. Moreover, by Theorem 4.7, $u$ is AMLE if and only if $u$ is AGME. In order to conclude the proof, it is sufficient to recall that AGMEs are infinity harmonic and, by the uniqueness results for the infinity Laplacian, the reverse implication holds in Riemaniann manifolds, Carnot groups and Grušin spaces.

Finally, we would like to apply our main theorem to a result recently proved by the first two named authors and Bieske in [6]. There, the authors have used the relation between AMLEs and infinity harmonic functions to give a geometric characterization of the set where the distance from a fixed point is infinity harmonic in Riemannian manifolds and Carnot groups. Actually, only one implication is necessary. Therefore that characterization can now be generalized to any subRiemannian structure. Let us briefly recall this result.

Let us fix a base point $x_{0} \in \mathbb{R}^{n}$ and define $d(x):=d\left(x, x_{0}\right)$ as, respectively, the Riemannian or Carnot-Carathéodory distance from $x_{0}$. Hereafter, we indicate by $E^{\prime}$ the set of all the points $x$ such that any geodesic from $x_{0}$ to $x$ ceases to be length minimizing exactly at $x$.

Theorem 4.11 ([6]). The function $d(x)$ is $A M L E$ in the set $A:=\mathbb{R}^{n} \backslash\left(E^{\prime} \cup\left\{x_{0}\right\}\right)$, since there $d(x)$ satisfies comparison by metric cones by above and below.

In Euclidean spaces $E^{\prime}=\emptyset$, thus the Euclidean distance $d(x)=|x|$ is infinity harmonic in $\mathbb{R}^{n} \backslash\{0\}$, as we already knew. In the Riemannian sphere $S^{n}$ we get that $d(x)$ is AMLE in $S^{n} \backslash\left\{x_{0}, x_{1}\right\}, x_{1}$ being the antipodal point to $x_{0}$. In the 1-dimensional Heisenberg group, the result implies that the Carnot-Carathéodory distance from the origin is AMLE outside the center of the group.

Applying Theorem 4.10 we get the following corollary.

Corollary 4.12. If $d(x)$ is a Riemannian or Carnot-Carathéodory distance from a point, then $d(x)$ is infinity harmonic in $A$. 
Proof. By Theorem 4.11 we know that $d(x)$ is AMLE in $A$. By Theorem 4.10 this implies that $d(x)$ is also infinity harmonic, in any Riemannian or sub-Riemannian structure. Hence the corollary is proved.

In [6] the authors have shown explicitly that $d(x)$ is not infinity harmonic in the center of the Heisenberg group.

The relation between so-called bilateral solutions of a Hamilton-Jacobi equation and the corresponding Aronsson equation found by Soravia in 23] can be applied to the special case of the eikonal equation and the infinity Laplace equation. In this case, Soravia's result yields the same characterization by geodesics. In fact it is known that the Carnot-Carathéodory distance from a point is a viscosity solution of the eikonal equation in $\mathbb{R}^{n}$ except at that point (see [9] and [19]) and by [23] it turns out to be a bilateral solution exactly in $A=\mathbb{R}^{n} \backslash\left(E^{\prime} \cup\left\{x_{0}\right\}\right)$. Therefore, at least in Carnot groups, the set $A$ is exactly the set where $d(x)$ is AMLE and infinity harmonic.

Proving the same characterization in general sub-Riemannian structures seems harder, due in particular to the difficulties concerning comparison principles for the infinity Laplace equation in such a generality.

\section{REFERENCES}

[1] V. Arnold, Ordinary differential equations, Springer-Verlag, Berlin, 2006. MR 2242407 (2007b:34001)

[2] G. Aronson, M.G. Crandall, P. Juutinen, A tour of the theory of absolutely minimizing functions, Bull. Amer. Math. Soc. 41 (2004), no. 4, 439-505. MR2083637 (2005k:35159)

[3] F.H. Beatrous, T. Bieske, J. Manfredi, The maximum principle for vector fields, The $p$ harmonic equation and recent advances in analysis. Contemp. Math. 370, Amer. Math. Soc., Providence, RI, 2005, 1-9. MR2126697[(2005m:35021)

[4] A. Bellaïche, The tangent space in sub-Riemannian geometry, in Sub-Riemannian Geometry, (eds. J.J. Risler and A. Bellaïche), Birkhäuser, Basel, 1996, 1-78. MR1421822 (98a:53108)

[5] T. Bieske, Lipschitz extensions on generalized Grushin spaces, Michigan Math. J. 53 (2005), no. 1, 3-31. MR 2125531 (2006i:35032)

[6] T. Bieske, F. Dragoni, J. Manfredi, The Carnot-Carathéodory distance and the infinite Laplacian, J. Geom. Anal. 19 (2009), no. 4, 737-754. MR2538933 (2011b:53065)

[7] T. Champion, L. De Pascale, Principles of comparison with distance functions for absolute minimizers, Journal of Convex Analysis 14 (2007), no. 3, 515-541. MR.2341302 (2008j:49073)

[8] M.G. Crandall, L.C. Evans, R.F. Gariepy, Optimal Lipschitz extensions and the infinity Laplacian, Calc. Var. P.D.E. 13 (2001), no. 2, 123-139. MR.1861094 (2002h:49048)

[9] F. Dragoni, Metric Hopf-Lax formula with semicontinuous data, Discrete Contin. Dyn. Syst. 17 (2007), no.4, 713-729. MR.2276470 (2007k:35044)

[10] B. Franchi, P. Hajlasz, P. Koskela, Definitions of Sobolev classes on metric spaces, Annales de l'Institut Fourier 49 (1999), no. 6, 1903-1924 . MR 1738070(2001a:46033)

[11] N. Garofalo, D.-M. Nhieu, Lipschitz continuity, global smooth approximations and extension theorems for Sobolev functions in Carnot-Carathéodory spaces, J. Anal. Math. 74 (1998), 67-97. MR 1631642(2000i:46025)

[12] J. Heinonen, Calculus on Carnot Groups, Fall School in Analysis, Jyväskylä (1994), 1-31. MR.1351042 (96j:22015)

[13] R. Jensen, Uniqueness of Lipschitz Extensions: Minimizing the Sup Norm of the Gradient, Arch. Ration. Mech. Anal. 123 (1993), no. 1, 51-74. MR.1218686 (94g:35063)

[14] P. Juutinen, Absolutely minimizing Lipschitz extensions on a metric space, Ann. Acad. Sci. Fenn. Math. 27 (2002), no. 1, 57-67. MR1884349 (2002m:54020)

[15] P. Juutinen, N. Shanmugalingam, Equivalence of AMLE, strong AMLE, and comparison with cones in metric measure spaces, Math. Nachr. 279 (2006), no. 9-10, 1083-1098. MR 2242966 (2008e:31009) 
[16] E.J. McShane, Extension of range of functions, Bull. Amer. Math. Soc. 40 (1934), 837-842. MR.1562984

[17] R. Montgomery, A Tour of Subriemannian Geometries, Their Geodesics and Applications, American Mathematical Society, Providence, 2002. MR 1867362 (2002m:53045)

[18] R. Monti, M. Rickly, Geodetically convex sets in the Heisenberg group, J. Convex Anal. 12 (2005), no. 1, 187-196. MR2135806 (2005m:53045)

[19] R. Monti, F. Serra Cassano, Surface measures in Carnot-Carathéodory spaces, Rend. Mat. Acc. Lincei 9 (2001), no. 3, 155-167. MR.1865002 (2002j:49052)

[20] B. O'Neill, Semi-Riemannian geometry. With applications to relativity, Pure and Applied Mathematics, 103. Academic Press, Inc. [Harcourt Brace Jovanovich, Publishers], New York, 1983. MR719023 (85f:53002)

[21] P. Pansu. Métriques de Carnot-Carathéodory et quasiisométries des espaces symétriques de rang un, Ann. of Math. (2) 129 (1989), 1-60. MR979599 (90e:53058)

[22] Y. Peres, O. Schramm, S. Sheffield, D.B. Wilson, Tug-of-war and the infinity Laplacian, J. Amer. Math. Soc. 22 (2009), no. 1, 167-210. MR2449057(2009h:91004)

[23] P. Soravia, On Aronsson equation and deterministic optimal control, Appl. Math. Optim. 59 (2009), 175-201. MR2480779 (2009m:35167)

[24] V. S. Varadarajan, Lie groups, Lie algebras, and their representations, Springer-Verlag, New York, 1984. xiii+430 pp. MR.746308 (85e:22001)

[25] C. Wang, The Aronsson equation for absolute minimizers of $L^{\infty}$-functionals associated with vector fields satisfying Hörmander's condition, Trans. Amer. Math. Soc. 359 (2007), 91-113. MR2247884 (2007g:35021)

[26] H. Whitney, Analytic extension of differentiable functions defined in closed sets, Trans. Amer. Math. Soc. 36 (1934), no. 1, 63-89. MR:1501735

School of Mathematics, Cardiff University, Senghennydd Road, Cardiff, Wales, United Kingdom CF24 4AG

Department of Mathematics, University of Pittsburgh, Pittsburgh, Pennsylvania 15260

Dipartimento di Matematica, University of Padova, via Trieste 63, 35121 Padova, ITALY 